\title{
ATENCIÓN CLÍNICA CENTRADA EN LA PERSONA: PRINCIPIOS Y ESTRATEGIAS
}

\author{
Juan Enrique Mezzich 1,2,a , Alberto Perales $3,4, a$
}

\begin{abstract}
RESUMEN
El desarrollo de una atención clínica centrada en la persona se inscribe dentro de un movimiento programático internacional hacia una medicina centrada en la totalidad de la persona. Este movimiento, con amplias bases históricas, ha ido madurando desde 2008 a través de encuentros entre instituciones globales de salud, proyectos investigativos y publicaciones académicas. El presente artículo se propone elucidar los principios conceptuales de la medicina centrada en la persona (MCP) y delinear estrategias para su aplicación práctica en servicios de atención clínica. La consecución de los mencionados objetivos se ha basado en revisiones de la literatura, consultas internacionales y reflexión sobre índices y pautas obtenidos. Los principios identificados de MCP son los siguientes: compromiso ético, marco holístico, sensibilidad cultural, foco comunicativo y relacional, individualización de la atención clínica, base común entre clínicos, paciente y familia para el entendimiento diagnóstico y la acción terapéutica, organización de servicios integrados y centrados en las personas, y educación médica e investigación en salud centradas en la persona. Adicionalmente, en relación con cada principio, se delinean estrategias propicias para su implementación en la atención clínica. Se concluye que los principios y estrategias presentados son consistentes con sugerencias ofrecidas en la literatura y que pueden servir de base para el diseño de índices y escalas. Se propone su continuo refinamiento a través de futuros estudios internacionales y locales para clarificar los conceptos claves del movimiento y estrategias útiles en aplicaciones clínicas prácticas.
\end{abstract}

Palabras Clave: Atención al paciente, Principios morales, Instituciones de atención ambulatoria (fuente: DECS BIREME).

\section{PERSON CENTERED CLINICAL CARE: PRINCIPLES AND STRATEGIES}

\begin{abstract}
The development of person-centered clinical care is inscribed within an international programmatic movement towards a medicine focused on the totality of the person. This movement, with broad historical bases, has been maturing since 2008 through conferences among global health institutions, research projects and academic publications. This paper is aimed at elucidating the conceptual principles of person-centered medicine (PCM) and to delineate strategies for the practical application of such principles in clinical care services. The above objectives have been approached through literature reviews, international consultations, and reflections on the patterns and indications obtained. The principles identified for person-centered medicine are the following: Ethical commitment, holistic framework, cultural awareness and responsiveness, communication and relational focus, individualized clinical care, common ground among clinicians, patient and family for joint diagnostic understanding and shared decision making, person- and community-centered organization of integrated services, and person-centered medical education and research. Additionally, pertinent strategies have been delineated for the implementation of such principles in clinical care. The authors conclude that the presented principles and strategies are consistent with suggestions offered in the literature and may serve as bases for the design of indices and scales. Their continuous refinement is proposed through future international and local studies. to clarify the key concepts of the movement as well as strategies for their practical clinical application.
\end{abstract}

Key Words: Patient Care, Morals, Ambulatory Care Facilities, (Source: MeSH NLM).

\footnotetext{
Icahn School of Medicine at Mount Sinai. New York, EE.UU.

Colegio Internacional de Medicina Centrada en la Persona. New York, EE.UU.

Instituto de Ética en Salud. Lima, Perú.

Facultad de Medicina, Universidad Nacional Mayor de San Marcos. Lima, Perú.

Médico psiquiatra

Recibido: 09/09/2016 Aprobado: 23/11/2016
}

Citar como: Mezzich JE, Perales A. Atención clínica centrada en la persona: principios y estrategias. Rev Peru Med Exp Salud Publica. 2016;33(4):794-800. doi: $10.17843 /$ rpmesp.2016.334.2567 


\section{INTRODUCCIÓN}

El desarrollo de una atención clínica centrada en la persona está inscrito dentro del movimiento programático internacional dirigido a colocar a la persona total y su contexto, en el centro de la salud y como meta de los servicios de salud. En consecuencia, este artículo primero reseñará brevemente las bases de una medicina centrada en la persona y, luego, el proceso de desarrollo del correspondiente movimiento programático internacional.

Se delinearán los objetivos, métodos y resultados de un proyecto de elucidación de los conceptos claves que subyacen a la medicina centrada en la persona $y$ al subsecuente planteamiento de estrategias para la implementación de los principios enunciados. Se comentarán estos logros y planteamientos en el contexto de esfuerzos similares, se examinará su valor para la construcción de instrumentos evaluativos y se esbozarán futuros estudios que afiancen y refinen los planteamientos expuestos.

\section{BASES DE UNA MEDICINA Y SALUD CENTRADAS EN LA PERSONA}

El desarrollo de la medicina moderna ha facilitado importantes avances científicos en el entendimiento de las enfermedades y sus implicaciones para el diagnóstico y tratamiento, así como la prolongación de las expectativas de vida. Tal desarrollo moderno, al mismo tiempo, ha favorecido un reduccionismo conceptual, atención hiperbólica al órgano y a la enfermedad, superespecialización profesional, fragmentación de la atención clínica, conversión de los actos de servicio en productos vendibles ${ }^{(1,2)}$, trivializando la relación médicopaciente y distanciándola de la solidaridad y respeto por la dignidad humana, e interfiriendo con una vocación de servicio a las personas que necesitan ayuda.

En respuesta a estas limitaciones y desviaciones, ha surgido un movimiento internacional que busca repriorizar a la persona total como centro de la medicina y la salud en consonancia con las raíces más tempranas de la medicina, encontradas en las más antiguas civilizaciones asiáticas y helénicas, las cuales tendían a conceptualizar la salud amplia y holísticamente ${ }^{(3-5)}$. En esta misma línea, el concepto de salud en la medicina precolombina establece un balance fundamental entre las dimensiones física, social y espiritual de la persona, en la cual la moderación en la dieta, el ejercicio y el comportamiento adecuado son considerados esenciales para una vida saludable ${ }^{(6,7)}$. Estas nociones históricas se reflejan en la abarcativa definición de salud inscrita en la constitución de la Organización Mundial de la Salud como Un estado de completo bienestar físico, mental y social y no meramente la ausencia de enfermedad ${ }^{(8)}$. En el Perú, Honorio Delgado (9) puntualizó en El Medico, la Medicina y el Alma, lo siguiente: El positivismo, generalización abusiva de las ideas válidas solo en el dominio de las ciencias físicas, lleva a considerar al enfermo como un objeto material, una cosa y a la medicina como una pura ciencia o una mezcla de ciencia y técnica, por ende, impersonal y mecánica. Carlos Alberto Seguín ${ }^{(10,11)}$ propuso un cambio radical donde los médicos no sean "veterinarios de seres humanos" sino "hombres frente a hombres". Seguín enfatizó en su docencia médica la importancia esencial del vínculo humano con el paciente.

\section{DESARROLLO PROGRAMÁTICO DE LA MEDICINA CENTRADA EN LA PERSONA}

La conceptualización y desenvolvimiento de una medicina y salud centradas en la persona y la comunidad ${ }^{(12-15)}$ han ido madurando a través de las Geneva Conferences on Person Centered Medicine llevadas a cabo anualmente desde 2008 en colaboración con la Organización Mundial de la Salud, la Asociación Médica Mundial, el Consejo Internacional de Enfermeras y la Alianza Internacional de Organizaciones de Pacientes, y la compañía de una treintena de instituciones globales de salud, y de cuyo proceso ha emergido un Colegio Internacional de Medicina Centrada en la Persona. El sustento académico de este movimiento internacional se puede ilustrar con la revista International Journal of Person Centered Medicine, publicada en colaboración con la Buckingham University Press ${ }^{(16)}$ y un próximo libro de texto sobre Psiquiatría Centrada en la Persona publicado por Springer (17) así como, con el coauspicio de importantes universidades de Europa y las Américas a los eventos mencionados, y con la recientemente establecida Red Latinoamericana de Medicina Centrada en la Persona ${ }^{(18)}$ que en esfuerzo conjunto con la Academia Nacional de Medicina del Perú y otras Academias de Medicina de la Región viene realizando Jornadas Latinoamericanas en este campo.

Esta nueva iniciativa mundial articula la ciencia y el humanismo hacia una medicina de la persona (y su salud total, de la enfermedad a la calidad de vida), para la persona (promoviendo el cumplimiento del proyecto vital de cada quien), por la persona (cultivando al profesional de la salud como persona, con elevadas aspiraciones éticas y científicas), y con la persona (colaborando respetuosamente con la persona que se presenta en busca de ayuda) ${ }^{(13,19)}$. Se trata pues de una medicina donde la ciencia es instrumento esencial y el humanismo su esencia misma. 


\section{OBJETIVOS}

La información sobre precedentes históricos y retos clínicos y de salud pública anteriormente resumida provee indicaciones y perspectivas pertinentes a la conceptualización de la medicina centrada en la persona. Tal entendimiento puede optimizarse a través de esfuerzos hacia una sistematización de las nociones prevalentes y pertinentes. El Colegio Internacional de Medicina Centrada en la Persona (ICPCM) ha hecho suyo este empeño a través de presentaciones y discusiones en las Conferencias de Ginebra desde 2008 (20,21). Más recientemente, esta preocupación institucional del ICPCM se ha plasmado en un proyecto sobre la conceptualización sistemática de la atención centrada en la persona y la medición de avances en este campo contando con el apoyo de la Organización Mundial de la Salud ${ }^{(22)}$. Basándose en tal proyecto, este artículo se propone elucidar los principios conceptuales de la medicina centrada en la persona y luego delinear estrategias para su aplicación práctica en servicios de atención clínica.

\section{MÉTODOS}

La consecución de los mencionados objetivos se ha basado en revisiones de la literatura, consultas internacionales y reflexión sobre índices y pautas obtenidos. Estas aproximaciones se describen brevemente a continuación, en gran parte constituidas por la metodología del proyecto del ICPCM anteriormente mencionado. La presentación detallada del estudio del ICPCM y sus diferentes aspectos se hace en otra publicación ${ }^{(23)}$.

\section{REVISIÓN DE LA LITERATURA}

La revisión de la literatura en torno a las bases de la atención de salud centrada en la persona y la comunidad se llevó a cabo en dos fases. La primera se focalizó en las ponencias hechas en las Conferencias de Ginebra sobre Medicina Centrada en la Persona del 2008 al 2010, los artículos disponibles sobre esas ponencias en los archivos del Colegio Internacional de Medicina Centrada en la Persona, y la literatura adicional proveniente de miembros de grupos consultivos. La segunda fase involucró una búsqueda de los bancos de información de PubMed de la United States National Library of Medicine en años subsecuentes. Los resultados de la revisión de los 70 artículos encontrados pertinentes fueron tabulados para facilitar la identificación de patrones e índices. La información tabulada incluyó: autores y fecha, título de la publicación, sumario de resultados e ideas clave ${ }^{(23)}$.

\section{CONSULTA INTERNACIONAL}

Esta consulta involucró dos grupos internacionales amplios. El Grupo Central colaborando estrechamente con los directores del proyecto a través de teleconferencias estuvo conformado por 17 expertos de las Américas, Europa, África, Asia y Oceanía, incluyendo a médicos de múltiples especialidades, así como representantes de enfermeras, asistentes sociales, pacientes y familiares. El segundo grupo involucró a un panel amplio constituido por 56 expertos internacionales provenientes de todos los continentes, incluyendo a médicos y otros profesionales de la salud, tanto clínicos como investigadores. Este panel fue consultado a través de correos electrónicos.

La primera fase de la consulta internacional involucró la discusión y evaluación de la revisión tabulada de la literatura por el Grupo Central. La tarea asignada a este grupo fue identificar en la literatura tabulada un conjunto de áreas claves descriptivas de servicios de salud centrados en la persona y la comunidad. Este conjunto incluyó un primer grupo de 14 áreas relacionadas con "Atención Personal a la Salud" y otro de 7 áreas relacionadas con "Salud Pública y Organización de Servicios". Más específicamente, el primer grupo de áreas lucía centrado en la persona, mientras que el segundo lucía centrado en la comunidad. A continuación, las áreas obtenidas de la literatura fueron organizadas en un formulario para facilitar su examen y procesamiento por el Panel Amplio. La sección final del formulario ofrecía espacio para listar áreas conceptuales adicionales extraídas de la literatura por los panelistas mismos. Se solicitó también a los panelistas calificar la importancia (alta, media, o baja) de cada una de las áreas listadas para describir atención centrada en la persona, así como delinear dentro de cada área elementos cruciales para caracterizar atención centrada en la persona y la comunidad.

La siguiente fase de la consulta internacional involucró el análisis, por el Grupo Central, de los formularios evaluativos producidos por el panel amplio con cara a diseñar en colaboración con los directores del estudio un Índice de Atención Centrada en la Persona (Personcentered Care Index, $\mathrm{PCl}$ )

\section{REFLEXIÓN HACIA ESTRATEGIAS PRÁCTICAS}

Los directores del estudio ICPCM y más recientemente los autores de este artículo llevaron a cabo sesiones de reflexión para ponderar los resultados de las consultas y el emergente índice de atención centrada en la persona. Su objetivo fue identificar estrategias prometedoras 
para la implementación de los principios de medicina centrada en la persona hacia una atención clínica centrada en la persona. El coautor de este artículo tradujo y adaptó lingüísticamente el Índice al español para su uso en Latinoamérica.

\section{RESULTADOS}

\section{FORMULACIÓN DE PRINCIPIOS DE MEDICINA CENTRADA EN LA PERSONA}

Sobre la base de la referida revisión de la literatura, consultas internacionales y reflexión ponderada, los siguientes ocho conceptos clave o principios de medicina centrada en la persona fueron identificados:

- Compromiso ético.

- Sensibilidad y respuesta cultural.

- Marco holístico.

- Foco comunicativo y relacional.

- Atención individualizada.

- Base compartida para el entendimiento diagnóstico y toma de decisiones.

- Organización de servicios integrados y centrados en la comunidad.

- Educación e investigación centradas en la persona.

Cada uno de estos conceptos clave alberga una serie de denotaciones y connotaciones que ayudan a explicar su significado, implicancias yalcances. Provienen del proceso de revisión de la literatura, las consultas internacionales y la reflexión ponderada. Su especificación detallada ha sido presentada por Mezzich JE et al. ${ }^{(23)}$.

\section{DISEÑO DE UN ÍNDICE DE ATENCIÓN CENTRADA EN LA PERSONA}

Los directores del estudio del ICPCM con los comentarios de Grupo Central de consultantes internacionales antes mencionado se abocaron a diseñar un índice prototipo que permita la evaluación de progreso hacia una atención centrada en la persona y la comunidad. Con este objetivo, se utilizaron los ocho conceptos clave aceptados como índices mayores. Se consideró que los seis primeros correspondían a atención clínica individual, mientras que los dos últimos se referían a sistemas generales de salud y a sus actividades de apoyo respectivamente. Los conceptos clave o índices mayores se encontraron relacionados con un conjunto de subconceptos que sumaban 33 subíndices. Se postuló que la presencia de cada uno de estos índices y subíndices en un determinado servicio o sistema de salud podría calificarse en términos de su frecuencia usando una escala de 4 puntos (1=Nunca, $2=A$ veces,
3=Frecuentemente, y 4=Siempre). Además, se propuso que para lograr un puntaje promedio global, se sumen los puntajes parciales y divida el resultado entre el número de ítems que habían podido ser evaluados. Se acordó también disponer de un espacio en blanco al final del formato para comentarios narrativos adicionales.

La Tabla 1 muestra la versión en español del Personcentered Care Index (PCI) (Índice de Atención-Centrada en la Persona).

\section{ESTRATEGIAS PRÁCTICAS PARA LA ATENCIÓN CENTRADA EN LA PERSONA}

La formulación de estrategias prácticas para la atención centrada en la persona como implementación de los principios de la medicina centrada en la persona reveló, también, una serie de conceptos más específicos de naturaleza denotativa o connotativa que fueron aprovechados para el diseño del índice de atención centrada en la persona. De este proceso y la reflexión ponderada mencionada anteriormente, surgen una serie de propuestas como estrategias prácticas para la atención centrada en la persona. Estas se presentan, a continuación, en la Tabla 2, anidadas bajo los conceptos claves o principios de medicina centrada en la persona postulándose que pueden facilitar su implementación práctica.

\section{DISCUSIÓN}

Tanto los conceptos clave cuanto las estrategias prácticas presentadas en este artículo son consistentes con observaciones y sugerencias en la literatura reciente. Por ejemplo, Leyns \& De Maeseneer (25) obtuvieron resultados similares particularmente aplicados a la atención primaria de salud. Una encuesta internacional realizada por Harding ${ }^{(26)}$ sobre atención centrada en la persona mostró que esta perspectiva está ampliamente presente en las políticas de salud de la mayor parte de países anglo-parlantes, aunque con gran retraso en su implementación.

También se nota una sustantiva consistencia entre los conceptos y estrategias listadas en este artículo con los campos informativos y aspectos procesales del modelo de Diagnóstico Integral Centrado en la Persona (PID en sus siglas en inglés) ${ }^{(27)}$. En efecto, sus componentes incluyen claramente la mayoría de los principios y estrategias arriba mencionados. El modelo PID se ha aplicado en la Guía Latinoamericana de Diagnóstico Psiquiátrico, Versión Revisada (GLADP-VR) ${ }^{(28)}$, cuyo uso en la región se viene incrementando. Ya la primera edición de la Guía Latinoamericana (GLADP) ${ }^{(29)}$ había sido favorablemente comparada con el DSM-IV de la Asociación Psiquiátrica Americana y la Clasificación 
Tabla 1. Índice de atención centrada en la persona

\begin{tabular}{|c|c|c|c|c|c|}
\hline $\mathrm{N}^{\circ}$ & Indicadores & Nunca & A veces & Frecuentemente & Siempre \\
\hline 1. & Compromiso ético & & & & \\
\hline 1.1 & $\begin{array}{l}\text { Se respeta la dignidad de las personas involucradas (pacientes, familia, } \\
\text { clínicos, etc.) }\end{array}$ & 1 & 2 & 3 & 4 \\
\hline 1.2 & Se respetan los derechos del paciente & 1 & 2 & 3 & 4 \\
\hline 1.3 & Se apoya la autonomía del paciente & 1 & 2 & 3 & 4 \\
\hline 1.4 & Se promueve el empoderamiento del paciente & 1 & 2 & 3 & 4 \\
\hline 1.5 & $\begin{array}{l}\text { Se comprenden y respetan los valores, preferencias y necesidades personales } \\
\text { del paciente. }\end{array}$ & 1 & 2 & 3 & 4 \\
\hline 1.6 & $\begin{array}{l}\text { Se alienta y posibilita la realización del proyecto de vida del paciente } \\
\text { (propósitos, planes, "sueños") }\end{array}$ & 1 & 2 & 3 & 4 \\
\hline 2. & Conciencia cultural & & & & \\
\hline 2.1 & Se reconocen la identidad étnica y los valores culturales del paciente. & 1 & 2 & 3 & 4 \\
\hline 2.2 & $\begin{array}{l}\text { Se consideran activamente el lenguaje, las necesidades de comunicación y } \\
\text { preferencias del paciente }\end{array}$ & 1 & 2 & 3 & 4 \\
\hline 2.3 & Se aceptan y respetan el género y preferencias sexuales del paciente. & 1 & 2 & 3 & 4 \\
\hline 2.4 & Se da significativa consideración a las necesidades espirituales del paciente. & 1 & 2 & 3 & 4 \\
\hline 3. & Marco holístico & & & & \\
\hline 3.1 & $\begin{array}{l}\text { Se consideran los factores biológicos, psicológicos, sociales, culturales y } \\
\text { espirituales de la salud para la comprensión cabal del caso }\end{array}$ & 1 & 2 & 3 & 4 \\
\hline 3.2 & $\begin{array}{l}\text { Se presta atención tanto a la salud negativa (problemas de salud, } \\
\text { discapacidades) como a la salud positiva o bienestar (desempeño, resiliencia, } \\
\text { fortalezas, recursos y calidad de vida). }\end{array}$ & 1 & 2 & 3 & 4 \\
\hline
\end{tabular}

4. Énfasis relacional

4.1 Los profesionales de la salud, pacientes y familias trabajan en equipo.

4.2 Se establece empatía en la comunicación clínica.

4.3 Se fomenta la confianza interpersonal a través del proceso de atención de

4.3 salud.

5. Atención individualizada

5.1 La individualidad y particularidades del paciente se consideran para

5.1 fundamentar la atención.

5.2 El contexto histórico-social del paciente es sopesado en el proceso de atención de salud.

5.3 Se promueven el desarrollo personal y madurativo del paciente.

6. Bases compartidas para el diagnóstico y el planeamiento terapéutico

6.1 El diagnóstico del estado de salud, experiencia y factores contribuyentes

6.1 resulta de una comprensión entre clínicos, paciente y familiares

6.2 El diagnóstico es colaborativamente elaborado tomando en cuenta la persona total y la salud integral.

6.3 Las decisiones del plan de atención son tomadas colaborativamente.

7. Sistemas de atención integrados y centrados en la comunidad

7.1 Se promueve la salud y los derechos de todos en la comunidad.

7.2 La comunidad participa en la planificación de los servicios de salud.

7.3 Se promueve la colaboración interdisciplinaria y entre programas en todos los niveles de la organización de servicios.

7.4 Los servicios personalizados se orientan al logro de alta calidad y excelencia.

7.5 Los servicios de salud responden a las necesidades y expectativas específicas de la comunidad.

7.6 Los servicios de salud se integran y coordinan alrededor de las necesidades de los pacientes.

7.7 Los servicios enfatizan la atención primaria centrada en la comunidad.

7.8 Los servicios aseguran la continuidad de la atención.

7.9 Los servicios se apoyan en las perspectivas internacionales sobre atención centrada en la persona.

$\begin{array}{llll}1 & 2 & 3 & 4 \\ 1 & 2 & 3 & 4 \\ 1 & 2 & 3 & 4\end{array}$

8. Educación e investigación centradas en la persona

El sistema de salud promueve la educación en salud pública centrada en la

8.1 persona.

8.2 El sistema de salud promueve el adiestramiento profesional centrado en la persona.

8.3 El sistema de salud promueve la investigación clínica centrada en la persona.

Puntaje global promedio:

Comentarios evaluativos adicionales: 
Tabla 2. Principios y estrategias para una atención clínica centrada en la persona

\begin{tabular}{|c|c|}
\hline $\begin{array}{l}\text { Principios de Medicina } \\
\text { Centrada en la Persona }\end{array}$ & $\begin{array}{l}\text { Estrategias para una atención clínica } \\
\text { centrada en la persona }\end{array}$ \\
\hline Compromiso ético & $\begin{array}{l}\text { Respeto a la dignidad de la persona } \\
\text { Reconocimiento de la autonomía y responsabilidad de la persona en el cuidado de su salud } \\
\text { Consentimiento informado como proceso ético dialógico } \\
\text { Promoción de su proyecto de vida. }\end{array}$ \\
\hline $\begin{array}{l}\text { Sensibilidad y respuesta } \\
\text { cultural }\end{array}$ & $\begin{array}{l}\text { Conciencia sobre la diversidad cultural (ancestro y contexto actual) con la que el paciente se } \\
\text { identifica } \\
\text { Atención y respeto por las explicaciones culturales del paciente sobre su salud y enfermedad } \\
\text { Conciencia de los clínicos sobre su propia identidad cultural } \\
\text { Respuesta integrativa en el diagnóstico y planes terapéuticos a las identidades culturales del } \\
\text { paciente y los clínicos }\end{array}$ \\
\hline Marco holístico & $\begin{array}{l}\text { Atención a los aspectos biológicos, psicológicos, sociales, económicos, ecológicos, culturales y } \\
\text { espirituales de la enfermedad } \\
\text { Atención a tales aspectos respecto de la salud positiva y el bienestar } \\
\text { Atención específica al contexto familiar en el entendimiento del estado de salud de la persona y en } \\
\text { acciones restaurativas y promocionales de la salud. } \\
\text { Consideración de la integridad total e interactivamente dinámica de la persona en su contexto }\end{array}$ \\
\hline $\begin{array}{l}\text { Foco comunicativo y } \\
\text { relacional }\end{array}$ & $\begin{array}{l}\text { Establecer empatía como sustento comunicacional clave } \\
\text { Facilitar que el paciente exprese todo lo que quiera expresar } \\
\text { Escuchar al paciente atentamente, con "más de dos oídos" [24] sintonizados con su subjetividad y } \\
\text { narrativa conscientes y subconscientes. } \\
\text { Consideración de la relación ética de servicio entre clínico y paciente. } \\
\text { Cultivar comunicación y relaciones efectivas con la familia y el equipo de profesionales } \\
\text { involucrados. }\end{array}$ \\
\hline Atención Individualizada & $\begin{array}{l}\text { Consideración del particular perfil biológico, psicológico y social de la persona } \\
\text { Consideración de sus factores de riesgo y factores protectores de salud } \\
\text { Consideración de su experiencia, valores y preferencias } \\
\text { Delineamiento de un programa individualizado para su atención }\end{array}$ \\
\hline $\begin{array}{l}\text { Base compartida para el } \\
\text { entendimiento diagnóstico y } \\
\text { las acciones terapéuticas }\end{array}$ & $\begin{array}{l}\text { Crear en cada caso una matriz colaborativa entre clínicos involucrados, paciente y familiares } \\
\text { Evaluación y formulación diagnóstica como entendimiento dialogal y mancomunado } \\
\text { Toma compartida de decisiones terapéuticas } \\
\text { Conducción de las acciones de salud con un sentimiento de responsabilidad compartida }\end{array}$ \\
\hline $\begin{array}{l}\text { Organización de servicios } \\
\text { integrados y centrados en la } \\
\text { comunidad }\end{array}$ & $\begin{array}{l}\text { Identificación de problemas y necesidades de salud de la comunidad } \\
\text { Planeamiento, desarrollo e implementación de servicios en colaboración con la comunidad } \\
\text { Establecimiento de mecanismos comunitarios para el monitoreo y seguimiento de servicios } \\
\text { Integración entre servicios de salud y sociales en la comunidad }\end{array}$ \\
\hline $\begin{array}{l}\text { Educación médica e } \\
\text { investigación de salud } \\
\text { centradas en la persona }\end{array}$ & $\begin{array}{l}\text { Atención al desarrollo personal, y no solo profesional, del estudiante, del profesor, y del } \\
\text { investigador } \\
\text { Investigación científica de la persona total y no solo de su enfermedad. } \\
\text { Consideración de la participación de la persona en las diferentes fases de las investigaciones } \\
\text { científicas } \\
\text { Fortalecimiento de políticas y mecanismos organizacionales para el cultivo de la ética en } \\
\text { instituciones académicas e de investigación. }\end{array}$ \\
\hline
\end{tabular}

Internacional de Enfermedades de la Organización Mundial de la Salud al ser considerada más holística y culturalmente sensible por psiquiatras de la región ${ }^{(30)}$.

Los principios de medicina centrada en la persona presentados en este artículo, están también dando lugar a la elaboración de instrumentos de medición como el Personcentered Care Index ${ }^{(23)}$ y su aplicación en la evaluación de servicios de salud centrados en la persona ${ }^{(31)}$.

En todo caso, el valor de las estrategias prácticas para una atención clínica centrada en la persona propuesta en este artículo deben ser documentadas a través de evaluaciones empíricas. Se recomiendan tales estudios tanto localmente como internacionalmente.

\section{CONCLUSIONES}

Los principios y estrategias listados parecen promisores para clarificar tanto los conceptos clave de la medicina centrada en la persona, como para desarrollar estrategias útiles en aplicaciones clínicas prácticas. A modo de síntesis y tomando en consideración los desarrollos conceptuales expuestos en este artículo, y sus perspectivas prácticas, puede decirse que la medicina centrada en la persona propone una atención clínica informada por la evidencia, la experiencia y los valores, y dirigida a la restauración y promoción de la salud y el bienestar de la persona total.

Conflictos de interés: los autores revelan ausencia de conflictos de interés. 


\section{REFERENCIAS BIBLIOGRÁFICAS}

1. Heath I. Promotion of disease and corrosion of medicine. Can Fam Physician. 2005; 51:1320-2.

2. Miles A. On a Medicine for the Whole Person: a way from scientistic reductionism and towards the embrace of complex clinical practice. J Eval Clin Pract. 2009; 15(6): 941-949.

3. Patwardhan B, Warude D, Pushpangadan P, Bhatt N. Ayurvedic and traditional Chinese medicine: a comparative overview. Evidence-based Complementary and Alternative Medicine. 2005; 2: 465-473.

4. Christodoulou GN. Psychosomatic Medicine. Plenum Press, New York, 1987.

5. Jouanna J. Hippocrates. Translated by M.B. Debevoise. Johns Hopkins University Press, Baltimore, 1999.

6. Anzures y Bolaños MC . Medicinas tradicionales y antropología. Anales de Antropología (I.I.A./U.N.A.M., México), 1978. 15: 151.

7. Mariátegui J.La concepción del hombre y de la enfermedad en el antiguo Perú. Rev. neuropsiquiatr. 1992; 55(3): 156166.

8. World Health Organization: Constitution of the World Health Organization. WHO, Geneva, 1946.

9. Delgado H. El Médico, la Medicina y el Alma. Ediciones Universidad Peruana Cayetano Heredia, Lima, 1992.

10. Seguin CA. La enfermedad, el enfermo y el médico. Ediciones Pirámide, Madrid, 1982.

11. Seguín CA. Tú y la Medicina. Editorial Poniente, Lima, 1993.

12. Mezzich JE. Psychiatry for the Person: Articulating Medicine's Science and Humanism. World Psychiatry. 2007; 6(2): 65-7.

13. Mezzich JE. Repensando el centro de la medicina: De la enfermedad a la persona. Acta méd. Peruana. 2010; 27(2): 148-150.

14. World Health Organization. Resolution on Primary Health Care, Including Health System Strengthening. In: Sixty-Second World
Health Assembly, Geneva, 18-22 May 2009. Resolutions and Decisions. Geneva, 2009 (WHA62/2009/ REC/1), Page 16.

15. World Health Organization. Global Strategy for People-centered Integrated Health Services. World Health Organization, Geneva, 2015.

16. Miles A, Mezzich JE. Advancing the global communication of scholarship and research for personalized healthcare: The International Journal of Person Centered Medicine. International Journal of Person Centered Medicine 2011; 1: 1-5.

17. Mezzich JE, Botbol M, Christodoulou GN, Cloninger CR, Salloum IM. Person Centered Psychiatry. Cham (Switzerland): Springer; in press.

18. Wagner P, Perales A, Armas R, Codas O, de los Santos R, Elio-Calvo D, et al. Bases y perspectivas latinoamericanas sobre medicina y salud centradas en la pesona. An. Fac. med. 2015; 76 (1): 63 70

19. Mezzich JE, Snaedal J, van Weel C, Heath I. The International Network for Person-centered Medicine: background and first steps. World Med Journal 2009;55:104-7.

20. Mezzich JE, Snaedal J, vanWeel C, Heath I. Conceptual Explorations on Person-Centered Medicine. Int J Integr Care. 29;10:e02.

21. Mezzich JE: The Geneva Conferences and the emergence of the International Network of Person-centered Medicine. J Eval Clin Pract. 2011; 17(2): 333-6

22. Mezzich JE, Kirisici L, Salloum IM. ICPCM Project on the Systematic Conceptualization and Measurement of Person- and People-centered Care. Technical Report, International College of Person Centered Medicine, New York, 2014.

23. Mezzich JE, Kirisci L, Salloum IM, Adams N, Wallcraft J, Trivedi JK et al. Systematically Conceptualizing Person Centered Medicine and Development and Validation of a Measurement Index. International Journal of Person Centered Medicine, en prensa.
24. Seguín CA. El Quinto Oído. Segunda Edición. Instituto Carlos Alberto Seguín, Lima; 2007.

25. Leyns C, De Maeseneer J. Conceptualizing person- and peoplecenteredness in primary health care: A literature review. International Journal of Person Centered Medicine 3: 13-22, 2013.

26. Harding E. Global "state of play" of person-centered care. Paper presented at the $9^{\text {th }}$ Geneva Conference on Person Centered Medicine, Geneva, Switzerland, April 12, 2016.

27. Mezzich JE, Salloum IM, Cloninger CR, Salvador-Carulla L, Kirmayer L, Banzato CE, et al. Person-centered Integrative Diagnosis: Conceptual Bases and Structural Model. Can J Psychiatry. 2010; 55:701-8

28. Asociación Psiquiátrica de América Latina. Guía Latinoamericana de Diagnóstico Psiquiátrico, Versión Revisada (GLADP-VR). Sección APAL de Diagnóstico y Clasificación, Lima, 2013.

29. Asociación Psiquiátrica de América Latina. Guía Latinoamericana de Diagnóstico Psiquiátrico, (GLADP). Sección APAL de Diagnóstico y Clasificación, APAL, San Carlos de Sonora, Mexico, 2004.

30. Saavedra JE, Mezzich JE, Otero A, Salloum IM. The revision of the Latin American Guide for Psychiatric Diagnosis (GLADP) and an initial survey on its utility and prospects. International Journal of Person Centered Medicine 2: 214-221, 2012.

31. Kirisci L, Hayes J, Mezzich JE. Evaluation of person-centered health services. In: Mezzich JE, Botbol M, Christodoulou GN, Cloninger CR, Salloum IM (eds): Person Centered Psychiatry. Springer Verlag, Heidelberg and New York, en prensa.

Correspondencia: Prof. Juan E. Mezzich Direccion: Icahn School of Medicine at Mount Sinai, Fifth Ave \& 100 St., Box 1093, New York, New York 10029, USA.

Correoelectrónico:juanmezzich@aol.com 